\title{
Diabetic Foot: Clinical Presentations and Management Outcome.
}

\author{
Biswas $\mathrm{SK}^{1}$, Roy $\mathrm{SR}^{2}$, SubbrataSarker $\mathrm{S}^{3}$, Biplob MMR ${ }^{4}$, Delta $\mathrm{MM}^{5}$, \\ Rahman $M^{6}{ }^{6}$, Paul $A^{7}{ }^{7}$, Saha $M^{8}$
}

\begin{abstract}
The diabetic foot, the foot of diabetic patients with ulceration, infection and/ or destruction of the deep tissues associated with neurological abnormalities and various degrees of peripheral vascular disease in the lower limb is quite common. A descriptive, cross-sectional study was carried out with an objective of assessing the common presentations and management outcome of diabetic foot. The study was carried out in the Surgery Department of Mymensingh Medical College Hospital, Mymensingh with a conveniently selected 130 patients of diabetic foot according to inclusion and exclusion criteria. The study period was one year. Out of 130 cases, the highest number of patients 55(42.4\%) were in age group 50 to 59 years with a mean age of 60.1 years and standard deviation (SD) of 9.8 years. Majority of the patients $91(70.0 \%)$ were male. Of them, $83(91.2 \%)$ were smokers. Of $130,51(39.2 \%)$ patients were illiterate and $41(31.5 \%)$ were farmer. Majority $67(51.53 \%)$ patients had history of uncontrolled diabetes for $10-15$ years. Most of the patients, $97(74.6 \%)$ were presented with neuropathic ulcer, whereas $24(18.4 \%)$ ischemic ulcers and $9(7.0 \%)$ were infective ulcers, and majority of the lesions $49(37.7 \%)$ were located on toes. Highest number of patients, $44(33.8 \%)$ were treated conservatively, while $37(28.4 \%)$ were treated by debridement, dressing and resurfacing, $17(13.1 \%)$ were treated by incision and drainage, and $32(24.6 \%)$ patients were treated by amputation. Most of the patients, $108(83.1 \%)$ were cured, of them, $36(33.3 \%)$ were treated conservatively, while $31(28.7 \%)$ by debridement and dressing, $17(15.7 \%)$ were treated by toe disarticulation, $13(12.0 \%)$ by incision and drainage, $4(3.7 \%)$ by above knee amputation, $4(3.7 \%)$ by ray amputation and $3(2.8 \%)$ by below knee amputation. Illiterate male smokers in their 6th and 7th decades of life with uncontrolled diabetes are the victim of diabetic foot. Neuropathy, vasculopathy, infections and trauma are identified risk factors. Conservative treatment, debridement and dressing, off-loading, culture-guided antibiotics therapy and amputations are the most successful modalities.
\end{abstract}

CBMJ 2017July: Vol. 06 No. 02 P: 25-31

Key words: Diabetic foot, Off-loading, Amputation, Re-surfacing, debridement.

\section{Introduction}

Diabetes is an epidemic disease worldwide, making it one of the most challenging health problems in the 21st century. In United States, $11.3 \%$ of all people of age 20 years or older have diabetes. In Bangladesh, the prevalence of type 2 diabetes mellitus of people age 20 years and above is $11.2 \%^{[1]}$. Indian subcontinent has the highest number of diabetic patients ${ }^{[2]}$. Diverse complications arise with long standing, uncontrolled or poorly controlled diabetic patients.

The diabetic foot is defined as the foot of diabetic patients with ulceration, infection and/ or destruction of the deep tissues associated with neurological abnormalities and various degrees of peripheral vascular disease in the lower limb (WHO \& IWG on diabetic foot).
1. Dr. Suttam Kumar Biswas

Asst. Prof. Dept. of Surgery

Community Based Medical College Winnerpar, Mymensingh

2. Dr. Shilpi Rani Roy

Lecturer, Dept. of Pharmacology

Mymensingh Medical College,Mymensingh.

3. Dr. Subbrata Sarker

Junior Consultant, Dept. of Surgery

Fulbaria Upozilla Health Complex

4. Dr. Md. Mustafizur Rahman (Biplob)

Junior Consultant, Dept. of Surgery

Valuka Upozilla Health Complex.

5. Dr. Md. Mustafizur Rahman (Delta)

Junior consultant, Dept. of Surgery

Tarash upozilla health complex

6. Dr. Md. Mizanur Rahman

Junior consultant, Dept. of Surgery

Jhenaigati upozilla health complex.

7. Dr. Arup Roton Paul

Asst. Prof. Dept. of Biochemistry

Community Based Medical College, Mymensingh

8. Dr. Moloy Kumar Saha

Asst. prof. dept. of Orthopaedic Surgery

Mymensingh Medical College,Mymensingh.

*Address of correspondence

Mobile : 01711151452

E-mail: suttamkumarbiswas@gmail.com 
Diabetic foot is the most common complication of diabetes mellitus, and is greater than retinopathy, nephropathy, heart attack and stroke combined. A patient with diabetic foot have major negative effects on quality of life due to loss of mobility, loss of work and reduction of social activities ${ }^{[3]}$.

Diabetic foot in type 2 diabetes has male predominance $(66.0 \%)$ and most of the patients are overweight, hyperglycemic having $\mathrm{DM}$ for more than 10 years. Ulcer is the most common presentation of diabetic foot. Neuropathic ulcers occur in $78.4 \%$ patients and the rest of the $22.6 \%$ had neuroischaemic ulcers $^{[4]}$.Foot ulcers are common in diabetic patient with prevalence of $25.0 \%{ }^{[5]}$.

Foot problems are a threat to every person with diabetes. Several pathophysiological mechanisms may be involved in diabetic foot $^{[6]}$. Hyperglycemia, atherosclerosis, polyneuropathy, infections are predisposing factors for pathological changes in diabetic foot. Increased blood sugar acts as a good culture media for bacterial growth, altered neutrophil functions, defective protein synthesis. Hyperglycemia results in increased levels of sorbitol in the cell, which acts like an osmolyte, a competitive inhibitor of myoinositol uptake. Sorbitol is direct toxic to nerve fibre. This preferential shunting of glucose through the sorbitol pathway results in decreased mitochondrial pyruvate utilization and decreased energy production. This process is termed as hyperglycemia induced pseudohypoxia $^{[7]}$. Atherosclerosis is a significant factor of diabetic foot. Hypertension, hyperlipidemia, chronic kidney disease, peripheral vascular disease (PVD) in lower extremity are the most common factors associated with limb ulceration, gangrene, impaired wound healing and ultimately amputation $^{[8]}$. Peripheral neuropathy clearly renders the patient victim to unrecognized injury, which potentiates the risk of bacterial invasion and infection ${ }^{[9]}$. Staphylococcus aureusand Pseudomonas aeruginosa are the most common organisms responsible for diabetic foot infections ${ }^{[10]}$. Anaerobic organisms are common causes of diabetic foot infection, but the prevalence is less ${ }^{[11]}$. Proper management of diabetic foot should be by multidisciplinary team approach. It is important to identify the patients at risk and therefore a detailed study of the natural history of diabetic foot, the various clinical characteristics $^{[12]}$. Diabetic foot can be prevented. For the prevention of diabetic foot problems, good glycemic control, lifestyle modifications with proper feet cares like keeping their feet dry and clean while also keeping them moisturized and protection from injury that may cause infection. Treatment of underlying disease processes, ensuring adequate blood supply, local wound care, infection control and pressure offloading are the essential components of diabetic foot management. Surgical options for management are revascularization, lower limb amputation and postamputation rehabilitation $^{[13]}$. Diabetic ulcers tend to heal slowly, need intensive care, and healing can be complicated by infection and gangrene, leading to long-term in-hospital treatment and/or amputations ${ }^{[2]}$.

Outcome of treatment of diabetic foot depends on stage of presentation. Treatment outcome is better in early stage like cellulitis, superficial ulcer. Outcome becomes poor in late stage presentation like fullfoot ulceration, gangrene and bony involvement ${ }^{[13]}$. Diabetic foot ulcers are associated with a lower quality of life, which affects both physical and mental health. Last but not the least, even after full cure, diabetic foot problem is prone to recurrence and needs continued follow up for rest of life.

This study has been undertaken to investigate the clinical presentations of diabetic patients with foot problems as well as its management outcome which can proclaim the status of the prognosis of the clinical condition and the role of appropriate intervention on the healing or amputation of the limb. The study can determine the specific treatment modalities which can reduce the period of hospital stay, and reduce the economic, psychological and social burdens effectively and thus improve the quality of life. 


\section{Methodology}

This was a descriptive cross-sectional type of observational study. The study was conducted at the Department of Surgery, Mymensingh Medical College Hospital, Mymensingh. Duration of study was one year ranging from October 2014 to September 2015. Dibetic patients with foot problem admitted into Surgery department of Mymensingh Medical College Hospital, Mymensingh during the study period were the Study population. The study was carried out with a Sample size of 130. Purposive type of non-random sampling technique was followed for the selection of sampling unit.

\section{Selection of the patients:}

\section{Inclusion criteria:}

1) Type 2 diabetic patients with foot problems admitted into the different. Surgical units of Mymensingh Medical College Hospital during thisstudy period.

2) Age more than 40 years.

3) Both sex.

Exclusion criteria:

1) Patient with type-1 diabetes mellitus.

2) Patient with previous amputation.

Data were checked and edited for consistency. Data analysis done by SPSS software (version 18). The significance of this study tested statistically by using the appropriate tests.

Prior to commencement of this study, the thesis protocol was approved by the Ethical Committee of Mymensingh Medical College.

\section{Results}

A descriptive, cross-sectional type of observational study was conducted in Mymensingh Medical College Hospital from October 2014 to September 2015. The study was carried out among 130 patients with an objective to observe the clinical presentation of diabetes mellitus with foot problems and its management outcome.
Highest number of respondents 55(42.4\%) were in age group 50 - 59 years. Male female ratio was $2.3: 1$. Most of the patients $41(31.5 \%)$ were farmer, whereas $31(23.8 \%)$ were housewives, $23(17.7 \%)$ were service holder, $20(15.5 \%$ ) were businessmen, $7(5.4 \%)$ were rickshawpullers, $6(4.6 \%)$ were fishermen and $2(1.5 \%)$ patients were butchers. Regarding educational status, it was revealed that majority of the respondents $51(39.2 \%$ ) were illiterate. Out of 130 patients, $103(79.2 \%)$ had no or suboptimal knowledge about foot care, $83(63.8 \%)$ patients were smokers , $67(51.5 \%)$ were suffering from diabetes for $10-15$ years and $118(90.8 \%)$ had uncontrolled diabetes mellitus.91(70.0\%) patients had the history of trauma. $17(16.8 \%)$ were on insulin therapy.Regarding site of lession $49(37.7 \%)$ of lesions were located on toes, while $24(18.5 \%)$ were located on dorsum of the foot, $12(9.2 \%)$ on heel, $9(7.0 \%)$ on more than one locations, $8(6.1 \%)$ on the site of previous surgery, $5(3.8 \%)$ on malleolus and $4(3.0 \%)$ were located between toes. Out of 130 patients $66(50.8 \%)$ presented with superficial ulcer, while $19(14.6 \%)$ presented with gangrene of the foot, $17(13.1 \%)$ were presented with abscess, $15(11.5 \%)$ were presented with deep ulcer, $10(7.7 \%)$ were presented with cellulitis and $3(2.3 \%)$ respondents were presented with osteomyelitis. $57(43.8 \%)$ ulcers were neuropathy, whereas $40(30.8 \%)$ ulcers were neuroischemic and $24(18.4 \%)$ purely ischemic ulcers and the rest of the $9(7.0 \%)$ had infective ulcers. According to Doppler study dorsalispedis arterial flow was mild obstruction in $5(12.8 \%)$, moderate obstruction in $9(23.1 \%)$ and severe obstruction in $15(38.4 \%)$ cases. The flow in the posterior tibial artery was mild obstruction in $3(7.6 \%)$, moderate in $7(17.9 \%)$ and severe obstruction in $17(43.5 \%)$ patients. $104(80.0 \%)$ were hypertensive and $\mathrm{HbA} 1 \mathrm{c}$ of respondents were $95(73.1 \%)$ had above normal range and $35(26.9 \%)$ respondents had within normal limit.In accord to treatment, $44(33.8 \%)$ were treated conservatively, while $37(28.4 \%)$ patients were treated by debridement, dressing and resurfacing, $17(13.1 \%)$ patients were treated by incision and drainage, $32(24.6 \%)$ patients were treated by different forms of amputation. Of 32 
amputed cases, 20(62.5\%) patients were treated with toe disarticulation, $5(15.6 \%)$ patients with ray amputation, $4(12.5 \%)$ patients with above knee amputation and $3(9.4 \%)$ patients treated with below knee. Most of the patients $108(83.1 \%)$ were cured, while $22(16.9 \%)$ were not cured. Of cured, of them $36(33.3 \%)$ were treated conservatively, while $31(28.7 \%)$ by debridement and dressing, $13(12.0 \%)$ by incision and drainage, $4(3.7 \%)$ by above knee amputation, $3(2.8 \%)$ by below knee amputation, $4(3.7 \%)$ by ray amputation and $17(15.7 \%)$ were treated by toe disarticulation. Out of $22(16.9 \%)$ incured, $8(36.4 \%)$ treated conservatively, $6(27.3 \%)$ by debridement and dressing, $4(18.2 \%)$ by incision and drainage, $1(4.5 \%)$ by ray amputation and $3(13.6 \%)$ were treated by toe disarticulation. The patients who were treated by above and below knee amputation all were cured.

All diabetic ulcer cases were treated in hospital within available facilities. Major ischemic cases and infective cases were referred to higher centre after preliminary treatment for short duration. Since it was difficult to eradicate the etiological factors of diabetic ulcers, treatment was protracted. Moreover, it was difficult to achieve and maintain healing without continued care. This demands proper hygiene and sound economic background.

Table I : Age distribution of study population.

\begin{tabular}{|c|c|c|}
\hline Age in Years & Frequency & Percentage \\
\hline $40-49$ & 18 & 13.8 \\
\hline $50-59$ & 55 & 42.4 \\
\hline $60-69$ & 41 & 31.5 \\
\hline $70-79$ & 16 & 12.3 \\
\hline Total & 130 & 100.0 \\
\hline
\end{tabular}

* Mean $(\bar{x})=60.1$ Years; Standard Deviation(SD)= 9.8 Years

Table II: Distribution by presentations of diabetic foot.

\begin{tabular}{|l|c|c|}
\hline Presenting features & Frequency & Percentage \\
\hline Superficial ulcer & 66 & 50.8 \\
\hline Gangrene & 19 & 14.6 \\
\hline Abscess & 17 & 13.1 \\
\hline Deep ulcer & 15 & 11.5 \\
\hline Cellulitis & 10 & 7.7 \\
\hline Osteomyelitis & 3 & 2.3 \\
\hline Total & 130 & 100.0 \\
\hline
\end{tabular}

Figure 1: Simple bar diagram showing different modalities of treatment

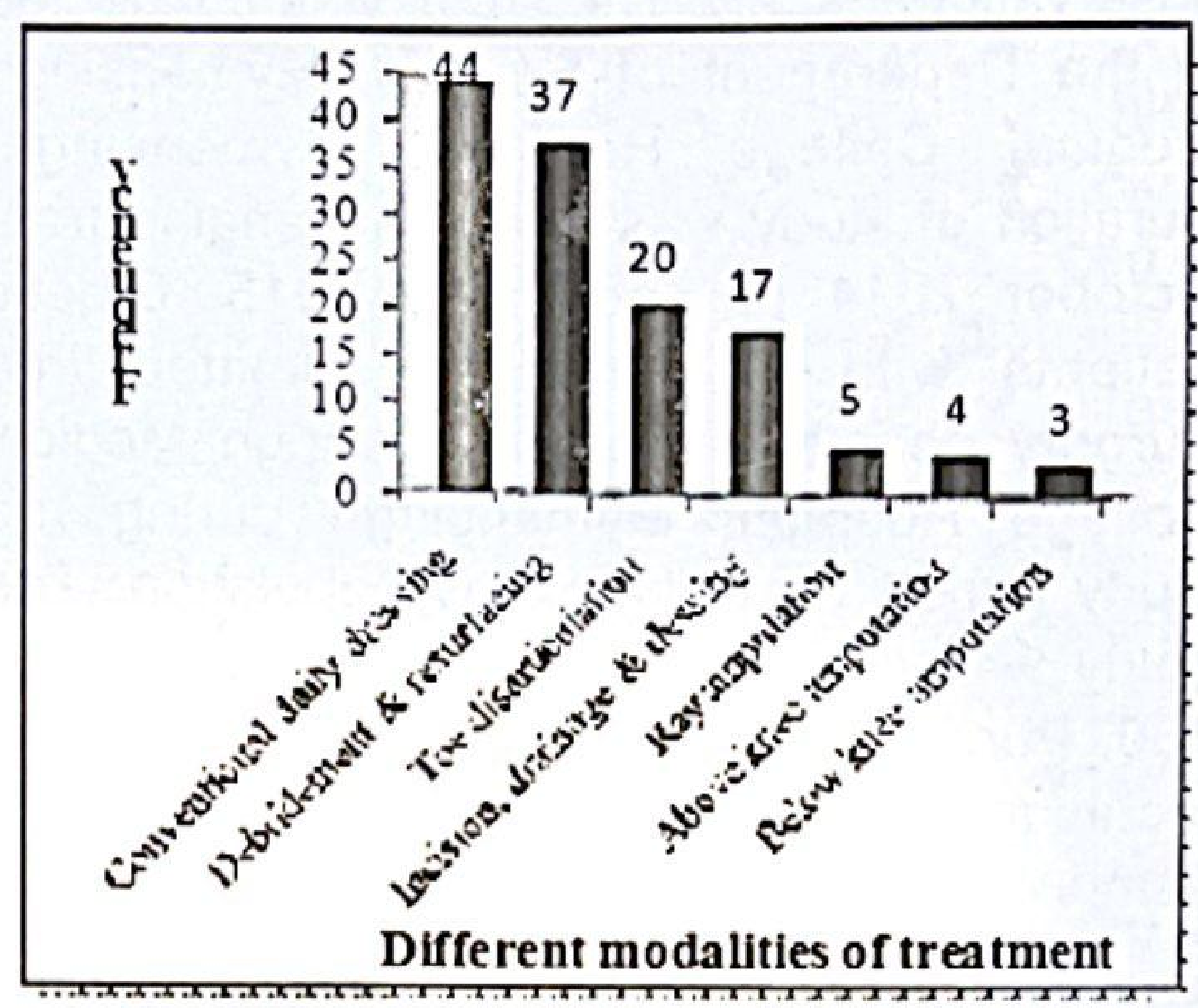

\section{Discussion}

A descriptive, cross-sectional type of observational study was carried out with a sample size of 130 in the Surgery Department of $\mathrm{MMCH}$ to assess the clinical presentations of diabetic foot and treatment outcome.

Age range of patients was $39(40-79)$ years with a mean age of 60.1 years and standard deviation (SD) of 9.8 years. Maximum number of patients $55(42.4 \%$ ) were within the age group of $50-59$ years. In other study, $56.3 \%$ were in the age group of 51-70 years, with mean age of 60.5 years and standard deviation (SD) of 9.9 years $^{[14]}$. These study findings are more or less consistent with the present study findings. Majority of the patients $91(70.0 \%)$ were male with a male-female ratio of $2.3: 1$. Diabetic foot ulcer was dominated by males $68.3 \%{ }^{[22]}$. At least $51(39.2 \%)$ patients were illiterate. These findings are more or less consistent with the findings of another study whereobserved that $28.6 \%$ of the patients were illiterate. It reflects that they are unaware of the complications of diabetes mellitus and its prevention as well[21]. Regardingoccupation, most of the respondents $41(31.5 \%)$ were farmer. These findings are in line with the findings of other study who observed that $43.8 \%$ patients were farmers. Most of the patients $79.2 \%$ had no or suboptimal knowledge about foot care. It 
indicates that they do not know about complications of diabetes as well as its preventive measures. It might be due to their illiteracy and unaviability of foot care education programme ${ }^{[16]}$. Maximum number $83(63.8 \%)$ patients were smokers. Another studyobserved that $87 \%$ of patients were smokers. Diabetic patients with habit of smoking are more to diabetic foot problems because of rapidly developing vasculopathy ${ }^{[22]}$. These findings substantiate to the present study findings. Duration of diabetes is directly related to the degree of wounds, while indirectly making the patient more vulnerable due to the complications of diabetes like nephropathy, neuropathy and retinopathy in the long run. Duration of diabetes in highest number of patients $67(51.53 \%)$ was over 10 years. In other study revealed that $48.54 \%$ had diabetes mellitus more than 10 years $^{[14]}$. Which is consistent with the present study findings. This findings indicate that more the duration of diabetes mellitus the more chance of developing foot problems. A highest majority of the patients $118(90.8 \%)$ had uncontrolled diabetes mellitus. This reflects that uncontrolled diabetic patients frequently develop foot ulcer $^{[16]}$. Minor trauma to the foot could not be recognized by the patients due to neuropathy, which creates portal of entry for microorganisms resulting foot ulcer. An overwhelming majority $91(70.0 \%)$ patients had the history of trauma. In other study observed that $65.0 \%$ patients had history of trauma ${ }^{[14]}$. This is in accord with the findings of the present study. The highest number $49(37.7 \%)$ of lesions were located on toes, while $24(18.5 \%)$ located on dorsum of the foot, $12(9.2 \%)$ on heel. In different study observed that $44.5 \%$ lesion located on toes, $14.2 \%$ on dorsum of foot, $10.9 \%$ on planter aspect, $4.6 \%$ on heel ${ }^{[9]}$. These findings are almost consistent with the present study findings. Majority patients $66(50.7 \%)$ were presented with superficial ulcer, while $19(14.6 \%)$ were presented with gangrene. In other study observed that $60.1 \%$ patients presented with superficial ulcer and $15 \%$ patients presented with gangrene ${ }^{[4]}$. These study findings are with more or less consistent with the present study findings.Ischaemia is generally associated neuropathy and neuroischaemic foot. The purely ischaemic foot without neuropathy is rarely seen in diabetic patients. Majority of the patients $97(74.6 \%)$ were presented with neuropathic ulceration, while $(30.7 \%)$ were with neuroischemic ulceration and $18.4 \%$ patients presented with purely ischaemic ulcer. Another study observed that $76.0 \%$ neuropathic ulcers and $57.2 \%$ neuroischaemic ulcers $^{[4]}$. These findings substantiate the present study findings. Most of the patients $104(80.0 \%)$ were hypertensive. Another study observed that $71.4 \%$ patients were hypertensive ${ }^{[21]}$. These findings are more or less consistent with that of the present study. HbA1c level that reflects the glycemic control status of previous three months. Majority 95(73.1\%) of patients had $\mathrm{HbA} 1 \mathrm{c}$ level above normal range. At least 120 patients had some sorts of associated complications. Of them, $97(74.5 \%)$ had neuropathy, $13(10.0 \%)$ had nephropathy, $8(6.0 \%)$ patients had retinopathy. Other study found that neuropathy in $75.0 \%$ cases $^{[13]}$. These findings are almost similar to the present study findings. Conservative treatment includes, antiseptic foot bath, regular meticulous dressing, antibiotics according to culture and sensitivity test, elevation of foot, improve nutrition and correction of anaemia. Highest number of patients $44(33.8 \%)$ were treated conservatively, while $37(28.4 \%)$ patients were treated by debridement, dressing and resurfacing, $17(13.1 \%)$ patients were treated by incision and drainage. As many as $32(24.6 \%)$ patients were treated by different forms of amputation. Of amputed patients, $20(62.5 \%)$ were treated with toe disarticulation, $5(15.6 \%)$ were treated with ray amputation, $4(12.5 \%)$ were treated with above knee amputation and $3(9.3 \%)$ were treated with below knee amputation. Other study observed that $30.2 \%$ smokers with diabetes mellitus needed amputation ${ }^{[18]}$. This findings is consistent with the present study findings. Outcome of treatment depends on patients age, nutritional status, presence of other comorbidities and facilities available. Out 
of 130 patients, $108(83.1 \%)$ were cured, while $22(16.9 \%)$ were treatment failed. Of cured $36(33.3 \%)$ were treated conservatively, while $31(28.7 \%)$ by debridement and dressing, $17(15.7 \%)$ by toe disarticulation, $13(12.0 \%)$ by incision and drainage, $4(3.7 \%)$ by above knee amputation, $4(3.7 \%)$ by ray amputation, and $3(2.8 \%)$ by below knee amputation. All the patients who were treated by above and below knee amputation were cured. In other study observed that $63 \%$ patients were successfully treated by conservative approach. However, treatment was failed in $37 \%$ cases. Twenty five percent patients treated by incision, debridement and dressing. Of them, in 19\% case treatment was failed. At least 13\% patients were treated by amputation ${ }^{[16]}$. These findings are inconsistent with present study findings. This might be due to small sample size and use of purposive sampling for the selection of patients.

\section{Conclusion}

Based on the study findings, it can be concluded that diabetic foot is common in illiterate male smokers in their 6th and 7th decades of life with uncontrolled diabetes for over 10 years. Moreover, patients with diabetic foot has inadequate knowledge about foot care, and afflicted with comorbidity like neuropathy. Neuropathy, vasculopathy, infections, trauma and occupations are identified risk factors for diabetic foot. Toes are the most common affected part. Staphylococcus aureus and Escherichia coli are the most common organisms involved in diabetic foot with multi-drug resistance. Conservative treatment, debridement and dressing, off-loading, culture-guided antibiotics therapy and amputations are the most successful treatment modalities.

Recommendation:

Based on study findings, following recommendations can be made:

1. Diabetic patient should be maintained optimum blood sugar and proper foot care.

2. Educating the diabetic patients to remain alert during nailparing, to keep protected from trauma, wearing protective shoes and to orient for self examination of the foot.

3. Orientation of medical and nursing personnel about diabetic foot care. Allocation of resources for training programs and management of diabetic foot.

4. "Multidisciplinary approach" for prevention and management of diabetic foot for improvement of quality of life that will reduce rate of amputation.

5. Conduction of a large scale study about diabetic foot for generalization of the findings.

\section{Limitations}

1. The study was of short duration.

2. This study was conducted only at one hospital with a small sample size and nonrandom sampling technique which limit the generalization of the findings.

\section{References}

1. Sayeed MA, Mahtab $H$, Khanam PA, Latif $Z A$, Banu A, Khan AK 'Prevalence of diabetes and impaired fasting glucose in urban population of Bangladesh'. Bangladesh Med Res Counc Bull.2007; 33(1):1-12.

2. Murugan $S$, Mani $K R$, Uma $D$ 'Prevalence of MRSA among diabetic patients with foot ulcers and their antimicrobial susceptibility pattern2008; 2:979-984.

3. Van Acker K, Leger P, Hartemann A, Chawla A, Siddiqui MK, (2014) 'Burden of diabetic foot disorders, guidelines for management, and disparities in implementation in Europe: a systematic literature review'. Diabetes Metab Res Rev.2014; 27.

4. Mehmood K, Akhtar ST, Talib A, Abbasi B, SirajU-Salekeen, Nagvi IH 'Clinical profile and management outcome of diabetic foot ulcers in a tertiary care hospital'. J Coll Physicians Surg Pak.2008; 18(7):408-412.

5. Jean-Louis Richard, Albert Sotto and JeanPhilippi Lavigne "New insights in diabetic foot infection'. World J Diabetes 2011; 2(2): 24-32.

6. Jeffcoate WJ, Chipchase SY, Ince $P$, Game $F L$, (2006) 'Assessing the outcome of the management of diabetic foot ulcers using ulcerrelated and person-related measures'2006; 2(9):1784-1787 
7. Cavanagh PR, Lipsky BA, Bradbury AW, Botek $G$ (2005), 'Treatment for diabetic foot ulcers'. Lancet 2005; 366 : 1725-1735

8. Gayle R, Benjamin AL, Gary NG 'The burden of diabetic foot ulcers'. The American Journal of Surgery 1998; 176 (Supp/ 2A):65-105.

9. Edmol ME, 'Experience in a multi disciplinary diabetic foot clinic'.1987;5(3) : 121-134.

10. Sharma VK, Khadka PB, Joshi A, Sharma $R$ 'Common pathogens isolated in diabetic foot infection'. Bir Kathmandu Univ Med $J$ (KUMJ)2006; 4(3): 295-301.

11. Abdulrazzak A, Bitar ZI, Al-Shamali AA 'Bacterological study of diabetic foot infections'. Diabetes Complications. 2005; 19(3) : 138-141.

12. Singh N, Armstrong DG, Lipsky BA 'Preventing foot ulcers in patients with diabetes'. JAMA.2005; 293:217-228

13. Wounds International-2013.

14. Bansal E, Garg A, Bhatia S, Attri AK, Chander $J$ 'Spectrum of microbial flora in diabetic foot ulcers'. Indian J Pathol Microbiol.2008; 5(1):204-208.

15. Bradbury A, Wilmink T, Lee AJ, Bell J, Prescott $R$, Gillespie I 'Bypass versus angioplasty to treat severe limb ischemia: factors that affect treatment preferences of UK surgeons and interventional radiologists'. J Vasc Surg.2004; 3(9):1026-1032.

16. Didier Pittet, MD, MS; BlaiseWyssa, MD; Catherine Herter-Clavel, MD; Karin Kursteiner, $M D$; Jean Vaucher, MD; P. Daniel Lew, MD 'Outcome of Diabetic Foot Infections, Treated Conservatively, A Retrospective Cohot Study With Long Term Follow-up'. 1999;159(8):851856.
17. International best practice guidelines: Wound management in diabetic foot ulcers. Wounds international, 2013.

18. International Working Group on the Diabetic Foot. International consensus on the diabetic foot and practical guidelines on the management and the prevention of the diabetic foot. Amsterdam: International Working Group on the Diabetic Foot, 2011.

19. K Bakkar, ZG Abbas, S Pendsey 'Step by Step, improving foot care in the developing world'. Practical diabetes Int.2006; 23(8): 365-369.

20. KatulandaP,RanasingheP, JayawardenaR, ConstantineGR, Sheriff MHR, Matthews DR 'The prevalence, patterns and predictors of diabetic peripheral neuropathy in a developing country'.2012; 4:21

21. Lee CM, Chang CC, Chen CM, Lai LJ, Chang $C F$, Chen $M Y$ 'The devil is in the detail: Prevention of diabetic foot ulceration in rural area is possible'.2013; 3:257-264.

22. Pandurengan $K$ 'Diabetic foot: Vasculopathy assessment and analysis of risk factors of amputation'. Int J Res Med Sci.2015; 3(1):7076

23. Wild S, Roglic G, GreenA, Sicree R, King $H$ 'Global prevalence of diabetes: Estimates for the year 2000 and projections for 2030'. Diabetes care.2004; 27:1047-1053. 